Trinity University

Digital Commons@ Trinity

Chemistry Faculty Research

Chemistry Department

4-28-2009

\title{
Adsorption of CO on Supported Gold Nanoparticle Catalysts: A Comparative Study
}

Heather Hartshorn

Trinity University

Christopher J. Pursell

TrinityUniversity, cpursell@trinity.edu

Bert D. Chandler

TrinityUniversity, bchandle@trinity.edu

Follow this and additional works at: https://digitalcommons.trinity.edu/chem_faculty

Part of the Chemistry Commons

\section{Repository Citation}

Hartshorn, H., Pursell, C. J., \& Chandler, B. D. (2009). Adsorption of CO on supported gold nanoparticle catalysts: A comparative study. Journal of Physical Chemistry C, 113(24), 10718-10725.

This Article is brought to you for free and open access by the Chemistry Department at Digital Commons @ Trinity. It has been accepted for inclusion in Chemistry Faculty Research by an authorized administrator of Digital Commons @ Trinity. For more information, please contact 


\title{
Adsorption of CO on Supported Gold Nanoparticle Catalysts: A Comparative Study
}

\author{
Heather Hartshorn, Christopher J. Pursell,* and Bert D. Chandler* \\ Department of Chemistry, Trinity University, San Antonio, Texas 78212-7200
}

Received: March 20, 2009; Revised Manuscript Received: April 28, 2009

\begin{abstract}
The adsorption of $\mathrm{CO}$ on three different gold nanoparticle catalysts supported on high surface area $\mathrm{TiO}_{2} \mathrm{was}$ studied using infrared transmission spectroscopy at room temperature and $\mathrm{CO}$ pressures typically used in $\mathrm{CO}$ oxidation reactions. The three, real-world catalysts were Au catalysts synthesized in our laboratory from thiol monolayer protected clusters (MPCs) and two commercial catalysts from the World Gold Council (WGC and AuTEK). Within experimental reproducibility, the adsorption data for the three catalysts are indistinguishable. While showing approximately Langmuir behavior, the adsorption data also show coverage dependence, as others have observed for many catalyst systems. Two approaches were used to fit the data, a two-site model and a variable binding constant model. The two-site Langmuir model yielded strong (36\%) and weak (64\%) binding constants of 2740 and $146 \mathrm{~atm}^{-1}$, respectively. Alternatively, using a sliding-tangent Langmuir fit gave a variable binding constant of $2670-120 \mathrm{~atm}^{-1}$ at room temperature for coverage $\theta=0-0.8$. The heat of adsorption was then extracted from the binding constants using a literature value for $-T \Delta S$. These values were determined as $\Delta H=-64$ and $-56 \mathrm{~kJ} / \mathrm{mol}$ for strong and weak binding according to the two-site model and $\Delta H=-63$ to $-56 \mathrm{~kJ} / \mathrm{mol}$ for coverage $\theta=0-0.8$ for the variable binding constant model. These values agree well with literature values obtained (i) using supported catalysts under higher pressures and (ii) using model catalysts under higher pressures and ultrahigh vacuum conditions.
\end{abstract}

\section{Introduction}

Supported Au catalysts are now well-established as the most active low-temperature CO oxidation catalysts known. ${ }^{1,2}$ Recent studies of gold and gold-based heterogeneous catalysts have extended the number of gold-catalyzed reactions to important reactions for industrial and synthetic chemists, including the water-gas shift reaction, ${ }^{3}$ alcohol $^{4}$ and alkene ${ }^{5}$ oxidations, hydrosilylation, ${ }^{6,7}$ the selective hydrogenation of nitroaromatics, ${ }^{8,9}$ and the production of commodity chemicals from biomass feedstocks. ${ }^{10}$ Indeed, the field of Au catalysis may be described largely in a regime of exploratory science, with numerous research groups working to discover new reactions and investigate new potential applications for Au-based materials.

Despite the tremendous research activity in gold-catalyzed oxidation reactions, the origins of the catalytic activity are still under discussion. One complicating factor is the wide range of reported activities, particularly in the case of $\mathrm{CO}$ oxidation. A recent review indicates that $\mathrm{CO}$ oxidation activities can vary by orders of magnitude for catalysts prepared in different laboratories using similar preparation techniques. ${ }^{2}$ However, to move from discovery-based science to deeper understanding, it is important to understand how catalysts differ and apply quantitative characterization techniques to compare catalysts prepared with different synthetic approaches.

To this end, carbon monoxide has long been used as a probe molecule for organometallic complexes, multimetallic metal clusters, and heterogeneous catalysts. ${ }^{11-15}$ The $\mathrm{CO}$ stretching frequency $\left(v_{\mathrm{CO}}\right)$ is remarkably sensitive to metal identity or electronic structure and can be used to rapidly evaluate the presence of metallic versus cationic surface metal atoms. It is also sensitive to adsorbate-adsorbate interactions. In the case

* To whom correspondence should be addressed. E-mail: Bert.chandler@ trinity.edu (B.D.C.), cpursell@ trinity.edu (C.J.P.). Phone: (210) 999-7557 (B.D.C.), (210) 999-7381 (C.J.P.). Fax: (210) 999-7569 (B.D.C.), (C.J.P.). of $\mathrm{CO}$ oxidation, the adsorption of $\mathrm{CO}$ is a likely fundamental step in the reaction mechanism and is, therefore, important to characterize in order to understand the catalysis.

Relative to other late transition metals, $\mathrm{CO}$ binding to the coinage metals is weak. ${ }^{16}$ Only a few reports have been able to determine the number of available CO binding sites on supported gold catalysts, albeit at substantially subambient temperatures. ${ }^{17,18}$ Consequently, there are relatively few infrared spectroscopic studies of CO adsorption on Au nanoparticles. Several studies have been performed on model catalysts under UHV conditions. Goodman's group examined $\mathrm{CO}$ adsorption on $\mathrm{Au}$ clusters deposited on $\mathrm{TiO}_{2} / \mathrm{Mo}(110)$ at low pressures and temperatures. ${ }^{19}$ They observed Langmuir-like adsorption on Au clusters with $d$ $=3.1 \mathrm{~nm}$, and isosteric plots yielded coverage-dependent heat of adsorption values. Behm's group also reported the adsorption of $\mathrm{CO}$ on $\mathrm{Au}$ clusters on $\mathrm{TiO}_{2} / \mathrm{Ru}(0001)$ under UHV conditions (low temperature and pressure), ${ }^{20}$ along with the more catalytically relevant conditions of $T=30-120{ }^{\circ} \mathrm{C}$ and $P_{\mathrm{CO}}=$ 7.5-37.5 Torr. ${ }^{21}$ Using a version of Temkin's adsorption model, they determined coverage-dependent heat of adsorption values that varied slightly with cluster size.

Work examining $\mathrm{Au}$ nanoparticles on various substrates includes a study of $\mathrm{CO}$ adsorption on Au clusters deposited on $\mathrm{FeO}(111) / \mathrm{Pt}(111)$. Lemire et al. reported size-dependent adsorption, which they attributed to the presence of highly uncoordinated gold atoms in very small particles. ${ }^{22}$ Finally, Yim et al. examined low-temperature and -pressure $\mathrm{CO}$ adsorption on $\mathrm{Au}$ clusters deposited on highly oriented pyrolytic graphite (HOPG) and on $\mathrm{Au}(111)$ roughened by argon-ion bombardment. ${ }^{23}$ They observed two TPD peaks with different heat of adsorption values, which they attributed to strong and weak binding. In particular, they noted the importance of low-coordinate steps and kinks in strongly binding $\mathrm{CO}$.

A number of UHV studies on unsupported Au surfaces similarly provide important background for the present work. 
Using a $\mathrm{Au}(110)-(1 \times 2)$ surface,${ }^{24}$ Goodman's group observed heat of adsorption values that depended on coverage and attributed the interactions to low-coordinate $\mathrm{Au}$ sites. Koel's group examined $\mathrm{CO}$ adsorption on $\mathrm{Au}(211)$ and reported heat of adsorption values for step and terrace sites. ${ }^{25}$ In particular, they observed low-coverage, strong non-Langmuirian binding on steps and higher-coverage, weaker Langmuirian binding on terraces.

Most of the infrared examinations of high surface area catalysts have involved single pressure measurements designed to characterize different catalysts. As such, there are relatively few pressure studies on high surface area catalysts, and few attempts have been made to determine the binding constant for $\mathrm{CO}$ adsorption. A recent study by Bianchi's group used infrared spectroscopy and relatively high pressures to measure the temperature dependence of isobaric $\mathrm{CO}$ adsorption on $\mathrm{Au}$ nanoparticle catalysts prepared via the deposition-precipitation method. ${ }^{26}$ By employing a version of Temkin's adsorption model, Bianchi's group determined heat of adsorption values that were coverage-dependent. Beyond this, however, we are aware of no other determination of the equilibrium constant for CO binding on high surface area gold catalysts, and we are unaware of any direct isothermal measurements.

Consequently, we set out to use infrared spectroscopy to measure $\mathrm{CO}$ adsorption on high surface area catalysts at room temperature. Importantly, because these catalysts generally work near room temperature with approximately $1 \% \mathrm{CO}$, we sought to develop a methodology that investigates the materials under conditions as close to the catalytic working conditions as possible. We recently reported on a method for preparing highly active model $\mathrm{Au}$ catalysts from thiol monolayer protected clusters (MPCs) and showed that our new catalysts were somewhat more active, but comparable to traditionally prepared materials. ${ }^{27}$ In the present study, we evaluate the fundamental step of $\mathrm{CO}$ adsorption on two commercial catalysts prepared via traditional means and compare them to the new bench scale catalyst. Our goals are to study $\mathrm{CO}$ adsorption as a fundamental step in $\mathrm{CO}$ oxidation catalysis and to compare $\mathrm{CO}$ adsorption on different catalysts to investigate its relative importance for influencing or predicting catalyst activity.

\section{Experimental Section}

Gold Nanoparticle Catalysts. The World Gold Council provided both the World Gold Council (WGC) and AuTEK catalysts. The WGC catalyst arrived as a powder and was used as received. The AuTEK catalyst arrived as ca. $5 \times 10 \mathrm{~mm}$ pellets. This catalyst is prepared with an "eggshell" structure (Au predominantly deposited on the top few micrometers of the pellet), so the surface of individual pellets was scraped with a spatula. The scrapings were then ground to a powder in an agate mortar and pestle.

Details of the synthesis of the thiol monolayer protected catalyst (MPC) have been previously reported. ${ }^{27,28}$ Briefly, Au nanoparticles were prepared in water using amine-terminated generation 5 polyamidoamine dendrimers (Dendritech). The nanoparticles were extracted into toluene as thiol monolayer protected clusters using decanethiol. After purification by multiple ethanol precipitations, the clusters were dissolved in methylene chloride and adsorbed onto the Degussa P-25 $\mathrm{TiO}_{2}$ support with stirring. Reduction under $\mathrm{H}_{2} / \mathrm{N}_{2}$ at $300{ }^{\circ} \mathrm{C}$ for $16 \mathrm{~h}$ has been shown to remove the thiols and produce active $\mathrm{CO}$ oxidation catalysts. $^{27}$

A complete structural and kinetic characterization of the MPC and WGC catalysts (transmission electron microscopy (TEM),

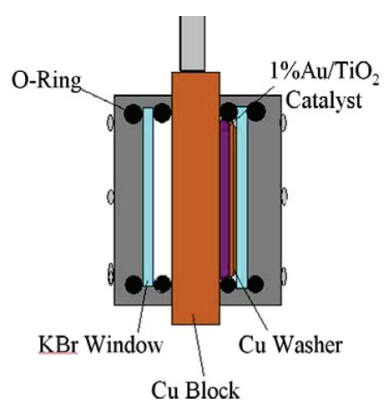

Figure 1. Cross section of the sample cell for infrared transmission spectroscopy of gold-supported catalysts. The $\mathrm{Au} / \mathrm{TiO}_{2}$ catalyst is pressed into a $30 \times 30 \mathrm{Ti}$ mesh and mounted inside the copper cell.

X-ray photoelectron spectroscopy (XPS), diffuse reflectance infrared Fourier transform spectroscopy (DRIFTS), kinetics of $\mathrm{CO}$ oxidation catalysis) has been reported previously. ${ }^{27}$ Importantly, the reduction treatment showed no observable changes in particle size for the WGC catalyst. Changes in Au particle size for the MPC catalyst were small and within the error of the TEM measurements; thus, this pretreatment does not appear to induce sintering for $\mathrm{Au} / \mathrm{TiO}_{2}$ catalysts. Accordingly, all catalysts underwent this reduction procedure prior to infrared spectroscopy measurements.

Infrared Spectroscopic Measurements. Catalyst samples for infrared analysis were prepared by pressing the $\mathrm{Au} / \mathrm{TiO}_{2}$ catalyst into a $30 \times 30 \mathrm{Ti}$ mesh (Unique Wire Weaving Co.). Most experiments were performed with approximately $20 \mathrm{mg}$ of catalyst, but other masses between 15 and $34 \mathrm{mg}$ were also used. The resulting mesh-supported pellet was placed in a tube furnace and heated overnight at $150{ }^{\circ} \mathrm{C}$. After cooling, the meshsupported pellet was mounted into a home-built copper cell and vacuum chamber; see Figure 1. This sample cell has a gasphase optical path length of $1 \mathrm{~cm}$. The entire vacuum chamber was placed in the sample compartment of a Nicolet Magna 550 FTIR spectrometer and evacuated to a pressure of $<1 \mathrm{mTorr}$ for $15 \mathrm{~min}$. All measurements were made at an ambient temperature of $297 \mathrm{~K}$. All spectra were referenced to a background spectrum of the $\mathrm{Au} / \mathrm{TiO}_{2}$ pellet under vacuum prior to the addition of CO. Transmission spectra consisted of 100 scans collected with $8 \mathrm{~cm}^{-1}$ resolution (spectral data spacing $=4 \mathrm{~cm}^{-1}$ ) and were reported in absorbance units.

The gas handling system consisted of a mechanical and diffusion pump, a glass line with stainless steel transfer lines to the sample apparatus, and a Baratron pressure gauge $(P=$ 0-10 Torr). A liquid nitrogen trap was used to trap out any impurities from the CO tank (UHP grade, from Air Products). The entire gas handling system was rinsed with $\mathrm{CO}$ three times before exposing the sample. After a background spectrum was collected, the sample was exposed to a low pressure of $\mathrm{CO}$ and the surface was allowed to equilibrate for 5-10 min. An infrared spectrum was recorded, and the pressure in the cell was slowly increased to the next pressure. After an experiment was completed, the sample was evacuated and the experiment repeated for a total of two or three adsorption isotherm measurements on a single catalyst sample in a single day.

\section{Results}

Three $\mathrm{Au} / \mathrm{TiO}_{2}$ catalysts (WGC, AuTEK, and MPC) were characterized by $\mathrm{CO}$ adsorption at room temperature using infrared transmission spectroscopy. Figure 2 shows the infrared spectra for a typical adsorption experiment for $\mathrm{CO}$ pressure from 0 to 10 Torr, in this case, using the World Gold Council (WGC) catalyst (34 mg pellet of $1 \% \mathrm{Au}$ on $\mathrm{TiO}_{2}$ ). Numerous control 


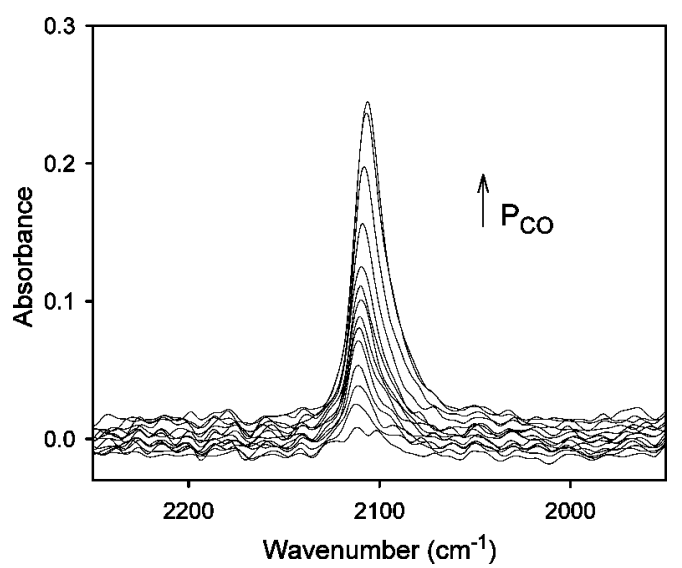

Figure 2. Infrared transmission spectra of $\mathrm{CO}$ adsorbed on the $\mathrm{Au}$ nanoparticle catalyst at room temperature $\left(1 \% \mathrm{Au}\right.$ on $\mathrm{TiO}_{2}$, WGC catalyst, $34 \mathrm{mg}$ pellet). $\mathrm{CO}$ pressure was increased from 0 to 10 Torr. Integrated peak area represents the $\mathrm{CO}$ coverage at each pressure. The $\mathrm{CO}$ peak shows a red shift with increasing coverage.

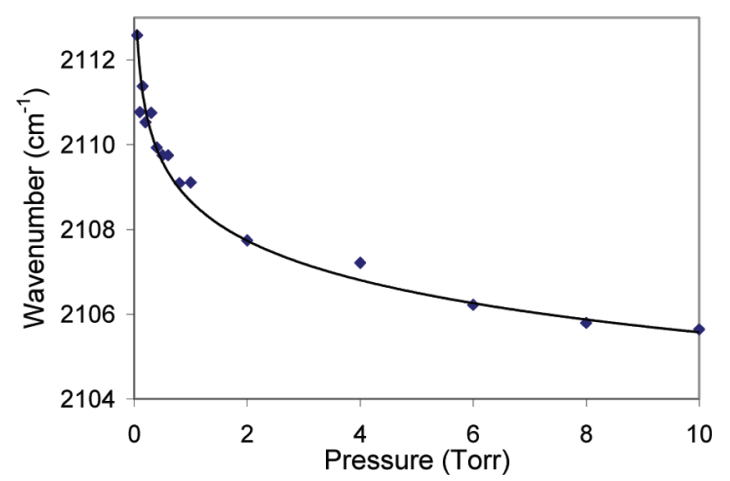

Figure 3. Infrared $\mathrm{CO}$ peak position showing a red shift as $\mathrm{CO}$ pressure increases. The smooth curve shown is to simply guide the eye.

experiments showed that the general features of the spectra were independent of the mass of catalyst used (only the peak area and maximum peak height changed). Additionally, the MPC and AuTEK catalysts had essentially the same spectra as those of the WGC catalyst, so plots similar to Figure 2 are omitted for brevity.

The infrared peak slightly above $2100 \mathrm{~cm}^{-1}$ is attributed to $\mathrm{CO}$ adsorption on $\mathrm{Au}$; no adsorption on $\mathrm{TiO}_{2}$ was observed at this temperature. ${ }^{29}$ The peak position shows a moderate red shift from 2113 to $2106 \mathrm{~cm}^{-1}$ as the $\mathrm{CO}$ pressure increases, shown in Figure 3. The data also suggest that some of the $\mathrm{CO}$ adsorption sites remain open at 10 Torr, as there is a continued red shift in the $\mathrm{CO}$ stretching frequency at the high end of this pressure regime. All three gold catalysts exhibited very similar infrared spectra and red shifts, so only the data from the WGC catalyst are presented. This shift is consistent with previously published results for $\mathrm{CO}$ adsorption on gold. ${ }^{19,22-25,30-34}$ The bonding between $\mathrm{CO}$ and $\mathrm{Au}$ is thought to occur through a combination of $\sigma$ and $\pi$ interactions. ${ }^{34}$ Briefly, the $5 \sigma$ bonding HOMO on $\mathrm{CO}$ donates into the metal s and d orbitals, stabilizing the HOMO and strengthening the $\mathrm{CO}$ bond. At the same time, the $2 \pi^{*}$ antibonding LUMOs withdraw some electron density from the metal d orbitals, weakening the $\mathrm{CO}$ bond. Increasing $\mathrm{CO}$ coverage leads to an increase in electron density at the metal surface, which decreases the extent of $5 \sigma$ donations from $\mathrm{CO}$ to Au. This, in turn, leads to a reduction in the stabilization of the $5 \sigma$ bonding orbital, which leads to a slightly weaker $\mathrm{CO}$ bond and the observed red shift in the $\mathrm{CO}$ vibrational peak position. $^{34}$

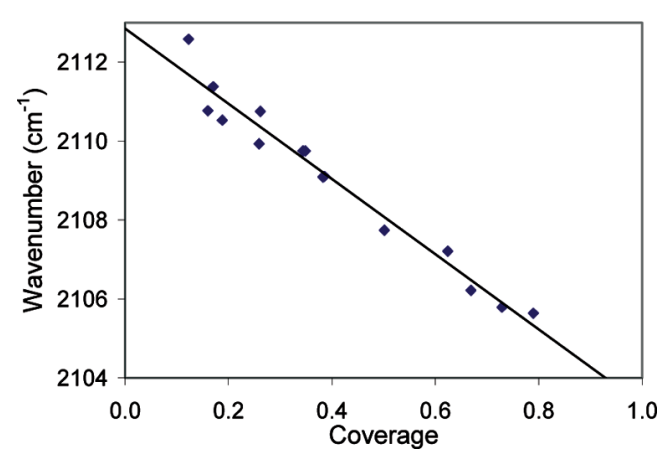

Figure 4. Infrared $\mathrm{CO}$ peak position showing a red shift with coverage. Coverage was determined from a Langmuir fit of the integrated peak area at higher $\mathrm{CO}$ pressures (see text and Figure 8). The line is a fit to the data, showing an approximately linear relationship.

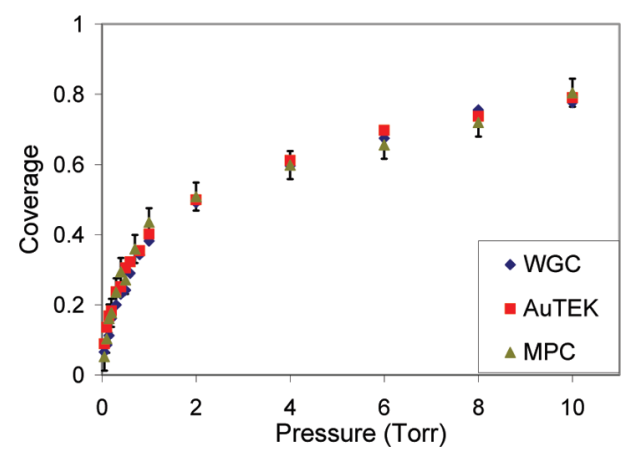

Figure 5. Langmuir isotherm plot for the three $\mathrm{Au}$ nanoparticle catalysts at room temperature. Coverage was determined from a Langmuir fit of the data at higher pressures (see text and Figure 8). Each point is the average of two to five individual experiments. Error bars representing one standard deviation are included for the MPC data. Within experimental reproducibility, all three catalysts exhibit similar coverage dependence.

The infrared data can be interpreted in terms of surface coverage (described in detail below) and are plotted in Figure 4. Extrapolation of this plot to $\theta=1$ yields a full-coverage $\mathrm{CO}$ stretching frequency of $2103.5 \mathrm{~cm}^{-1}$, in excellent agreement with measurements on model UHV systems ${ }^{19,22-25}$ and higherpressure studies with other supported Au catalysts. ${ }^{26}$

The integrated peak area $(S)$ was used to quantify the CO adsorption. The $S$ values for individual IR spectra were converted to $\mathrm{CO}$ coverage $(\theta)$ by normalizing to the maximum integrated peak area $\left(S_{\max }\right)$ for that particular isotherm adsorption experiment (vide infra). In this normalization, where $\theta=S / S_{\max }$, we examine only the coverage of $\mathrm{CO}$ binding sites and not necessarily the total coverage of the nanoparticle surface. The maximum peak area was determined from a Langmuir plot of the high-pressure data, as described below.

Figure 5 is a plot of coverage as a function of $\mathrm{CO}$ pressure for the three gold catalysts and shows that all three catalysts display very similar Langmuir-like behavior. Numerous control experiments using all the catalysts were performed, including experiments with differing pellet masses. In all cases, the normalized adsorption data showed essentially this same general behavior; hence, in Figure 5, we have presented averaged data from two to five individual isotherm experiments on each catalyst. Goodman's group also observed Langmuir-like behavior for the adsorption of $\mathrm{CO}$ on Au clusters deposited on $\mathrm{TiO}_{2} / \mathrm{Mo}(110) .{ }^{19}$ Their cluster size $(d=3.1 \mathrm{~nm})$ is very similar to the particle size of the catalysts of this study. 


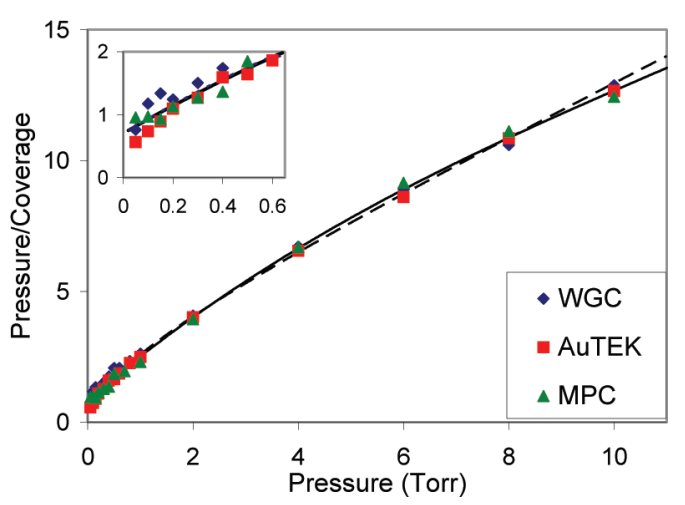

Figure 6. Room-temperature adsorption data for the three $\mathrm{Au}$ nanoparticle catalysts plotted over the entire pressure range in a linear form of the Langmuir equation. The data show a slight deviation from ideal Langmuir behavior over the large pressure range. A smooth, polynomial curve fit (solid line) has, therefore, been used to determine the binding constant as a function of coverage. Alternatively, the dashed line is a two-site model fit (see text).

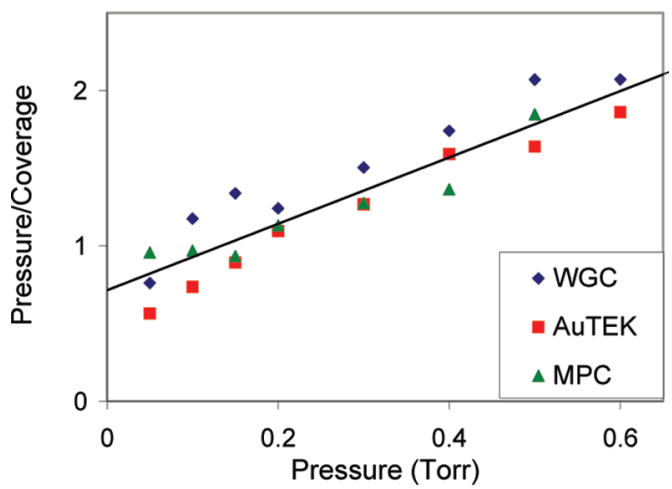

Figure 7. Lower-pressure adsorption data plotted in a linear form of the Langmuir equation. Within experimental reproducibility, the three catalysts have similar coverage dependence. The line is a fit of all data over this pressure range, demonstrating near-ideal Langmuir behavior.

The integrated infrared peak areas were, therefore, described using the following linear form of the Langmuir isotherm expression

$$
\frac{P}{S}=\frac{1}{K S_{\max }}+\frac{P}{S_{\max }}
$$

where $P$ is the $\mathrm{CO}$ pressure in Torr, $S$ is the $\mathrm{CO}$ integrated infrared peak area, $S_{\max }$ is the maximum peak area, and $K$ is the equilibrium constant for $\mathrm{CO}$ binding to the Au nanoparticle surface.

Figure 6 shows the adsorption data plotted according to the linear form of the Langmuir expression given in eq 1 . The data are not as linear as one might expect, and the solid curve included in the figure is a polynomial fit. This indicates that the adsorption process is more complicated than that of a singlesite adsorption model (the Langmuir isotherm carries an implicit assumption that all of the adsorption sites are equivalent). The data in Figure 6 can be split into two regions under low and high $\mathrm{CO}$ pressures, shown in Figures 7 and 8, respectively. For the lower $\mathrm{CO}$ pressure range (ca. $<1$ Torr), the $\mathrm{CO}$ adsorption data show near-Langmuir behavior and, within experimental scatter of the data, the three catalysts are essentially equivalent.

The data exhibit very good Langmuir behavior over the higher CO pressure range (ca. $4-10$ Torr). CO surface coverage, $\theta=$

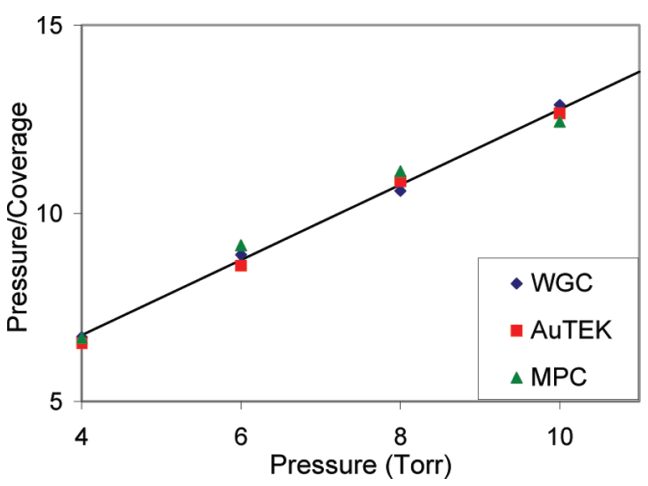

Figure 8. Higher-pressure adsorption data plotted in a linear form of the Langmuir equation. The line is a fit to all the data and has been used to convert the integrated infrared peak areas to coverage according to eq 1 (see text).

$S / S_{\max }$, was defined using $S_{\max }$ determined from the high CO pressure fit of eq 1, as shown in Figure 8. The high-pressure data are closest to $\theta=1$, which minimizes the error in the $S_{\max }$ value (determined from the slope of the line in Figure 8), giving what should be a reasonably precise value for $S_{\max }$. The value for $S_{\max }$ was subsequently used to normalize the infrared peak area data, converting the individual peak areas to $\mathrm{CO}$ coverages.

Figures 6-8 suggest that the adsorption data are best described by having a range of equilibrium constants (and, therefore, heats of adsorption) rather than a single binding constant. Coverage-dependent heat of adsorption is a wellknown phenomenon and has been observed for a number of metal-adsorbate systems. ${ }^{35-39}$ Several approaches can be used to describe the observed variation in $K$ and $\Delta H$ with coverage; we employed two straightforward treatments.

The first approach invokes a two-site model involving collections of strong and weak binding sites. Accordingly, the integrated infrared peak area is a measure of the total coverage of all CO binding sites (not all gold surface sites). At low CO pressures, the adsorption would be to the strong binding sites, as per Figure 7, whereas at high pressures, adsorption is to the weak sites, as per Figure 8. Thus, the observed variation with pressure of the binding constant can then be described as a net binding constant that arises from the relative population and availability of these two sites. The adsorption data can, therefore, be expressed by the following two-site Langmuir model

$$
\frac{S}{S_{\max }}=\frac{f_{\mathrm{s}} K_{\mathrm{s}} P}{\left(1+K_{\mathrm{s}} P\right)}+\frac{f_{\mathrm{w}} K_{\mathrm{w}} P}{\left(1+K_{\mathrm{w}} P\right)}
$$

where $f_{\mathrm{s}}$ and $f_{\mathrm{w}}$ are the fraction of strong and weak binding sites $\left(f_{\mathrm{s}}+f_{\mathrm{w}}=1\right)$ and $K_{\mathrm{s}}$ and $K_{\mathrm{w}}$ are the corresponding binding constants, respectively. Using SigmaPlot to fit the data to eq 2 yielded $K_{\mathrm{s}}=2740 \mathrm{~atm}^{-1}$ (36\% of the sites) and $K_{\mathrm{w}}=146 \mathrm{~atm}^{-1}$ $(64 \%)$. This fit is displayed in Figure 6 by the dashed curve through the data.

The second approach for accommodating the variation of $K$ and $\Delta H$ is to analyze the data only over very small pressure (i.e. coverage) ranges, where the values of $K$ and $\Delta H$ change only slightly. This can be done with a sliding-tangent fit to the Langmuir model shown in Figure 6. The data are fit to a polynomial function, and the derivative of that polynomial is calculated at discrete pressures to determine the slope of the curve $\left(1 / S_{\max }\right)$ at that particular pressure. Using the determined slope and the point at which it was calculated, we calculate the intercept of the line tangent to the curve $\left(1 / K S_{\max }\right)$ and use it to 


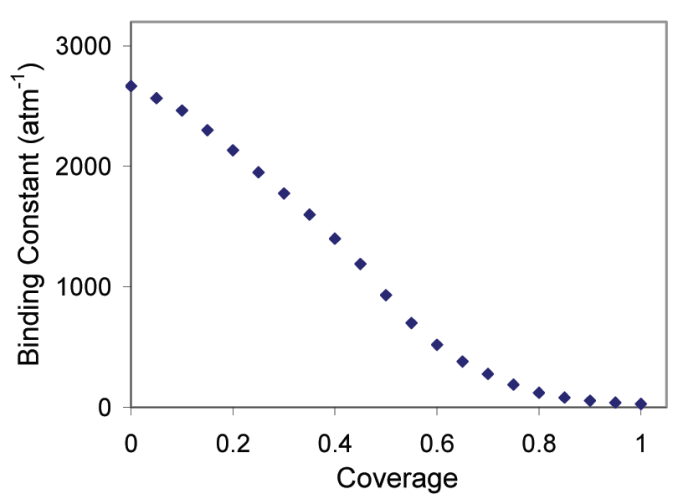

Figure 9. Binding constant for $\mathrm{CO}$ adsorption on $\mathrm{Au}$ nanoparticle catalysts at room temperature. The binding constant was determined according to eq 1 and the smooth curve in Figure 6. This analysis yields values of $\Delta G$ for $\mathrm{CO}$ adsorption that varies from -20 to $-8 \mathrm{~kJ} / \mathrm{mol}$ for low to high coverage, respectively. Since data were not collected for coverages above 0.8 , the binding constants above 0.8 are an extrapolation of the fit.

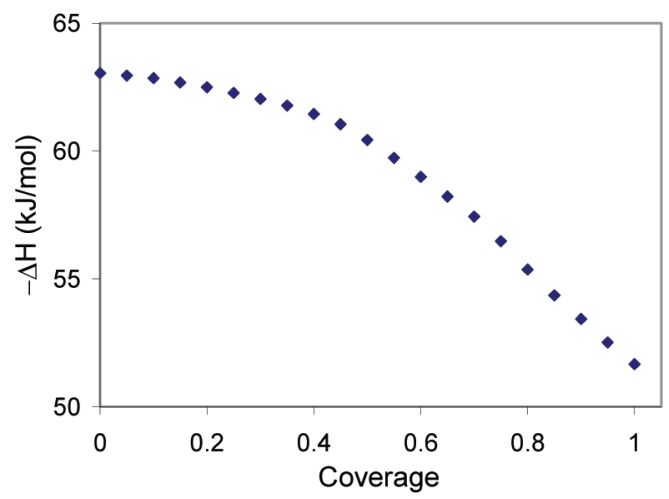

Figure 10. Enthalpy for $\mathrm{CO}$ adsorption on the three Au catalysts as a function of coverage. These values were determined from the binding constants at room temperature (see Figure 9) and a literature value for the entropy contribution, $T \Delta S=-44 \mathrm{~kJ} / \mathrm{mol}$ (see text).

extract the value of $K$ at that particular pressure (i.e., coverage), or $K=$ slope/intercept of eq 1 .

When we use this model, the binding constant for $\mathrm{CO}$ on $\mathrm{Au} / \mathrm{TiO}_{2}$ catalysts varies from 2670 to $120 \mathrm{~atm}^{-1}$ at room temperature for $\theta=0-0.8$, as shown in Figure 9. Since data were collected only to $\theta=0.8$, we have extrapolated out to $\theta$ $=1$, where $K=27 \mathrm{~atm}^{-1}$. Not surprisingly, the low- and highcoverage binding constant values agree with the two-site model's strong and weak binding constants.

From these $K$ values, $\Delta G$ at room temperature (ca. $297 \mathrm{~K}$ ) was determined as -20 and $-12 \mathrm{~kJ} / \mathrm{mol}$ for strong and weak binding sites, respectively, or as -20 to $-8 \mathrm{~kJ} / \mathrm{mol}$ over the range of $\theta=0-0.8$. When we used these $\Delta G$ values and a $T \Delta S$ entropy contribution of $-44 \mathrm{~kJ} / \mathrm{mol},{ }^{40}$ the $\Delta H$ of adsorption was determined to vary from -63 to $-52 \mathrm{~kJ} / \mathrm{mol}$, as shown in Figure 10 , or as -64 and $-56 \mathrm{~kJ} / \mathrm{mol}$ for strong and weak binding sites, respectively. To the best of our knowledge, this is the first time $K$ values have been reported for the adsorption of $\mathrm{CO}$ on actual $\mathrm{Au}$ catalysts under pressures comparable to those typically used in catalytic test reactions. As shown in Table 1 and discussed further below, these heat of adsorption values agree very well with previous determinations at higher pressures and on model systems.

\section{Discussion}

The working state of any catalyst is of fundamental interest and importance, so examining catalysts under conditions as near as possible to the working conditions is highly desirable. The weak binding of $\mathrm{CO}$ to Au nanoparticles has made examination of this important interaction difficult, so most quantitative studies have been carried out with low surface area model catalysts under UHV conditions. ${ }^{19,21,22,25}$ Studies with high surface area catalysts have been performed at much higher pressures, ${ }^{26,41}$ where $\mathrm{CO}$ induced reconstruction of the Au nanoparticles was observed. ${ }^{41} \mathrm{CO}$ oxidation catalysts are typically tested at or near ambient pressure, nominally using $1 \%$ CO (7-8 Torr) and between 0.5 and $20 \% \mathrm{O}_{2} \cdot{ }^{2}$ To our knowledge, the present study is the first to examine $\mathrm{CO}$ binding on high surface area supported $\mathrm{Au}$ catalysts under conditions similar to those of working catalysts.

The real-world catalysts examined in this study include bench (WGC) and industrial-scale (AuTEK) catalysts prepared by traditional methods as well as a catalyst prepared from thiolstabilized monolayer-protected clusters (MPC). The similarity in $\mathrm{CO}$ adsorption properties of the three catalysts is striking. Figure 5 shows that, within very reasonable errors, the three catalysts show essentially the same $\mathrm{CO}$ adsorption behavior. This is remarkable considering that the catalysts were prepared in three different laboratories, using vastly different preparation methods and production scales. It is even more surprising, in the context of the history of Au catalysis, where lab-to-lab reproducibility has only recently become common. ${ }^{2}$ Given the small differences in the $\mathrm{CO}$ adsorption properties of the three catalysts, $\mathrm{CO}$ binding and activation are probably not primary factors in differentiating the activity of these catalysts.

Determination of $\boldsymbol{K}$ and $\Delta \boldsymbol{H}$. To the best of our knowledge, binding constants for the adsorption of $\mathrm{CO}$ on gold nanoparticle catalysts have not been reported in the literature. ${ }^{1}$ Several groups have reported isobaric heat of adsorption measurements on $\mathrm{Au}$ nanoparticles, in which $\mathrm{CO}$ coverage was varied by changing the sample temperature (vida infra). These measurements have generally been made with model catalysts under UHV conditions or with high surface area catalysts using higher pressures. Our study is distinguished from these because we have made isothermal $\mathrm{CO}$ adsorption measurements on a variety of high surface area catalysts using pressures typically used in catalytic testing. This allows us to directly evaluate the equilibrium constant and extract the heat of adsorption to compare with literature measurements.

As shown in Figures 5-8, the three catalysts exhibit very similar near-Langmuir behavior. The slight deviation indicates that the adsorption process is more complicated than that of the single-site Langmuir adsorption model. However, without abandoning the simplicity of the Langmuir model, the data can be fit to modified expressions to determine experimental binding constants. The first approach is a two-site model, given by eq 2. As the dashed line through the data in Figure 6 shows, this simple model accurately represents the data. This model gives rise to two binding constants, $K=2740$ and $146 \mathrm{~atm}^{-1}$. It also provides an opportunity to estimate the relative number of strong (36\%) and weak (64\%) CO binding sites. Although this model may be an oversimplification, there are a number of literature reports describing two different $\mathrm{CO}$ adsorption regimes on model gold catalysts. ${ }^{24,25,42}$

Alternately, a Langmuir model with a coverage-dependent binding constant (and therefore binding energy) can be used to explain the differences in $\mathrm{CO}$ binding energy at low and high coverage. The fitting is accomplished using a sliding-tangent Langmuir expression, which also does a good job of describing the experimental data (the solid line through the data in Figure 6 nearly overlaps the two-site model fit). This model yields a 
TABLE 1: Heat of Adsorption for CO on Au Catalysts and Surfaces

\begin{tabular}{|c|c|c|c|c|c|c|c|}
\hline material & data analysis ${ }^{a}$ & $-\Delta H_{\mathrm{str}}{ }^{b}(\mathrm{~kJ} / \mathrm{mol})$ & $-\Delta H_{\mathrm{wk}}^{c}(\mathrm{~kJ} / \mathrm{mol})$ & $P$ (Torr) & $T(\mathrm{~K})$ & $\theta$ & ref \\
\hline $\mathrm{Au} / \mathrm{TiO}_{2}$ & 2-site Langmuir & 64 & 56 & $10^{-2}-10$ & 293 & $0-0.8$ & this work \\
\hline $\mathrm{Au} / \mathrm{TiO}_{2}$ & sliding tangent & 63 & 56 & $10^{-2}-10$ & 293 & $0-0.8$ & this work \\
\hline $\mathrm{Au} / \mathrm{TiO}_{2}$ & Temkin & 74 & 47 & $8-150$ & $125-400$ & $0-1$ & 26 \\
\hline $3 \mathrm{~nm} \mathrm{Au} / \mathrm{TiO}_{2} / \mathrm{Ru}(0001)$ & Temkin & 66 & 42 & $8-40$ & $303-493$ & $0.3-0.7$ & 21 \\
\hline $4 \mathrm{~nm} \mathrm{Au} / \mathrm{TiO}_{2} / \mathrm{Ru}(0001)$ & Temkin & 62 & 44 & $8-40$ & $303-493$ & $0.3-0.7$ & 21 \\
\hline $1.8 \mathrm{~nm} \mathrm{Au} / \mathrm{TiO}_{2} / \mathrm{Mo}(111)$ & $\mathrm{C}-\mathrm{C}$ & 69 & 63 & $10^{-8}-10^{-2}$ & $130-220$ & & 19 \\
\hline $\mathrm{Au}(110)-(1 \times 2)$ & $\mathrm{C}-\mathrm{C}$ & 45.6 & 32.6 & $10^{-8}-10^{-4}$ & $100-250$ & $0-0.3$ & 24 \\
\hline $\mathrm{Au} / \mathrm{TiO}_{2} / \mathrm{Ru}(0001)$ & TPD & 65 & 53 & & & & 20 \\
\hline $\mathrm{Au}(211)$ steps & TPD & 50 & & & & & 25 \\
\hline $\mathrm{Au}(211)$ terraces & TPD & 38 & 27 & & & & 25 \\
\hline roughened $\mathrm{Au}(111)$ & TPD & 54 & 44 & & & & 23 \\
\hline $\mathrm{Au} / \mathrm{HOPG}$ & TPD & 51 & 39 & & & & 23 \\
\hline
\end{tabular}

${ }^{a}$ Data based on infrared spectroscopy or temperature-programmed desorption (TPD) measurements. C-C is a Clausius-Clapeyeron treatment of infrared adsorption data. ${ }^{b}$ Heat of adsorption for strong $\mathrm{CO}$ binding sites (upper range of coverage-dependent heat of adsorption). ${ }^{c}$ Heat of adsorption for weak $\mathrm{CO}$ binding sites (lower range of coverage-dependent heat of adsorption).

range of binding constants that gradually decrease from $K=$ $2670 \mathrm{~atm}^{-1}$ at low coverage to $K=120 \mathrm{~atm}^{-1}$ at the highest coverage (ca. $\theta=0.8$ at 10 Torr); see Figure 9. This model is consistent with literature reports of coverage-dependent binding energies, even when particle sizes are varied under controlled UHV conditions. ${ }^{21,26}$

We are unaware of other researchers using a sliding-tangent Langmuir expression to evaluate adsorption data on gold catalysts. The values extracted from this model are consistent with the more traditional two-site model as well as with literature reports (vida infra). The two models are slightly different ways of interpreting the data and yield essentially the same low- and high-coverage binding constants. Although the two-site model is likely to be an oversimplification, it does provide some information regarding the range of binding constants and the relative number of strong and weak binding sites.

The sliding-tangent model, on the other hand, more accurately reflects the complexity of a supported catalyst, which has a range of particle sizes and interactions with the irregular support. It is a simple and straightforward model that allows the binding constant to vary smoothly with coverage. Because the data are fit to an arbitrary fitting function and changes in the slope of this function with pressure are evaluated, the sliding-tangent model does not rely on assumptions regarding the nature of the interaction and does not extensively use fitting parameters.

Our approaches can be compared with the more complicated Temkin isotherm analysis utilized by Bianchi ${ }^{26}$ and Behm. ${ }^{21}$ In that model, the binding energy (or heat of adsorption) is assumed to vary linearly from a low coverage $\left(E_{0}\right.$ at $\left.\theta=0\right)$ to a high coverage value ( $E_{1}$ at $\left.\theta=1\right)$. The adsorption coefficients $\left(K_{0}\right.$ and $K_{1}$, which are similar to binding constants) are determined using a statistical thermodynamics expression. Theoretical curves are then generated, and the $E_{0}$ and $E_{1}$ values (and, therefore, the $K_{0}$ and $K_{1}$ values) are adjusted until a good fit to the pressure-dependent coverage data at various temperatures is achieved. Although the Temkin analysis reproduces the adsorption data fairly well, it is rather complicated, assumes that the energy of adsorption varies linearly with coverage, and requires adsorption data at numerous temperatures.

Comparison to Literature Values. Table 1 is a compilation of the heat of adsorption values extracted from our measurements alongside previous measurements on other systems. It also includes some of the important experimental parameters. Only Bianchi's group has reported detailed CO adsorption measurements on high surface area $\mathrm{Au} / \mathrm{TiO}_{2}$ catalysts; ${ }^{26}$ all of the other measurements are on single-crystal model systems. Bianchi's group used pressures of 8-150 Torr and subsequently discovered that the higher $\mathrm{CO}$ pressures induced some sort of reconstruction of the $\mathrm{Au}$ nanoparticles. ${ }^{41}$ Typical reaction conditions are at 8 Torr or lower, so their measurements are at the upper boundary of those used in practice. Behm and coworkers used a slightly lower high-pressure range and were able to measure $\mathrm{CO}$ adsorption on different sized $\mathrm{Au}$ particles. ${ }^{21}$ Goodman's group has also reported size-dependent CO adsorption, but under more typical UHV conditions. Our measurements on high surface area catalysts nicely fit in the gap between Goodman's, Behm's, and Bianchi's measurements.

Table 1 shows that our data fall well within the range of previous measurements. Our coverage-dependent $\Delta H$ values are bounded by Bianchi's measurements on similar materials and are very similar to both Behm and Goodman's measurements. This indicates that, at least for $\mathrm{CO}$ adsorption measurements on this system, the pressure and material gaps are not significant issues. The only real difference between our values and those reported by others is the range between the high and low coverage values. Our range is about $10 \mathrm{~kJ} / \mathrm{mol}$ (which is similar to some of Goodman's ranges), whereas others typically report a range of $20 \mathrm{~kJ} / \mathrm{mol}$ or more.

Koel's values are obtained from TPD measurements and, therefore, do not provide the same sort of coverage-dependence information. ${ }^{25}$ They are, however, an important benchmark because they provide data for adsorption on the well-defined $\mathrm{Au}(211)$ surface. ${ }^{25}$ Our strong binding sites (as our Bianchi's, Goodman's and Behm's) are more exothermic than Koel's measurements on $\mathrm{Au}(211)$ surfaces. This suggests that more highly uncoordinated $\mathrm{Au}$ corner and edge atoms are responsible for the strong $\mathrm{CO}$ binding, as others have concluded. ${ }^{2}$ The lower range of our data approaches Koel's values, so surface arrangements resembling $\mathrm{Au}(211)$ surfaces may be involved in the weaker binding sites.

Implications for CO Oxidation Catalysis. Evaluating the current results based on previous $\mathrm{CO}$ oxidation studies with these catalysts and the context of the body of catalysis literature may also shed some light onto the important factors for the high activity of $\mathrm{Au} / \mathrm{TiO}_{2}$ catalysts. We recently reported an in-depth study of the preparation of the MPC catalyst as well as CO oxidation kinetics for both the WGC and MPC catalysts. ${ }^{27}$ XPS and elemental analysis showed no residual sulfur on the MPC 
catalyst after our activation protocol. Indirect measurements (IR, XPS, and TEM studies) were essentially identical for the WGC and MPC catalysts and showed no substantial differences between the two catalysts. Within very good experimental errors, the two catalysts have the same Arrhenius apparent activation energy and nearly identical reaction orders in both $\mathrm{O}_{2}$ and $\mathrm{CO}$. The only measurable difference between the two catalysts is the slightly higher activity of the MPC catalyst (ca. $40 \%$ faster rates per total $\mathrm{Au}$ ), which was attributed to the preparation of a greater number of active sites when the preformed nanoparticles were used as catalyst precursors.

Three important conclusions regarding the reaction mechanism on working catalysts can be drawn from the current data: (i) the $\mathrm{CO}$ binding sites are likely to be under-coordinated corner and edge atoms (vide supra), (ii) these binding sites are not saturated with $\mathrm{CO}$ under typical reaction conditions, and (iii) oxygen, which binds much more weakly to $\mathrm{Au}$ than does $\mathrm{CO}$ (vida infra), can effectively compete with $\mathrm{CO}$ for low-coordination $\mathrm{Au}$ atoms. The directly observable red shift in $\mathrm{CO}$ stretching frequency (Figures 2-4) and the normalized peak area data (Figures 5 and 6) show that the catalysts are not saturated with $\mathrm{CO}$ under catalysis conditions (nominally 7-8 Torr). The important conclusion from this data is that, even at extremely low conversions $(<1 \%)$ during catalysis, a substantial fraction of the $\mathrm{CO}$ adsorption sites (roughly $25 \%$, see Figure 5) remain unoccupied. ${ }^{43}$ In terms of a reaction mechanism, the presence of free $\mathrm{CO}$ binding sites means that $\mathrm{CO}$ desorption, which is inherently an endothermic process, is not required to open up a site for oxygen coordination. With more traditional Pt and Pd catalysts, $\mathrm{CO}$ binds too strongly and essentially covers all of the surface atoms, leaving few, if any, available sites for $\mathrm{O}_{2}$ binding and activation. ${ }^{44}$

Our data probe the energetics of the interaction between $\mathrm{CO}$ and the Au surface and provide insight into the fraction of open $\mathrm{CO}$ binding sites; however, the data are only semiquantitative. We cannot evaluate the total number of $\mathrm{CO}$ binding sites, and it is important to clarify that these measurements are not equivalent to a chemisorption experiment. The data provide no direct information regarding the total number of $\mathrm{CO}$ binding sites, and it cannot be inferred that three-quarters of the total surface is covered by $\mathrm{CO}$ at 8 Torr. Our data only indicate that roughly $25 \%$ of the $\mathrm{CO}$ binding sites are available. Since these are likely to be under-coordinated corner and edge atoms, this is likely to be a small fraction of the total $\mathrm{Au}$ surface atoms.

Indeed, CO chemisorption values for supported Au catalysts have been notoriously difficult to measure, and researchers have often resorted to indirect methods to evaluate the number of active sites. For example, Oxford and co-workers found that intentionally poisoning an $\mathrm{Au} / \mathrm{TiO}_{2}$ catalyst with 5-10 mol \% bromide completely shuts down the catalytic activity. ${ }^{17} \mathrm{We}$ are aware of only one report of CO chemisorption in the literature, using low-temperature pulse chemisorption techniques. ${ }^{18} \mathrm{Me}$ negazzo and co-workers' measurements on $\mathrm{Au} / \mathrm{TiO}_{2}$ catalysts, which included measurements on a sample of the WGC catalyst, indicated that roughly $3 \%$ of the total $\mathrm{Au}$ atoms bind $\mathrm{CO}$ at temperatures between 150 and $180 \mathrm{~K}$. Their average particle sizes were between 3 and $4 \mathrm{~nm}$, so this is a small fraction of the total surface atoms (around 10\%). On the basis of a structural model, the authors concluded that $\mathrm{CO}$ bound to about one of every three step-edge sites on the catalyst. Thus, the total number of $\mathrm{CO}$ adsorption sites is likely to be only a fraction of the low-coordinated corner and edge atoms. ${ }^{18}$

Recent theoretical work suggests that the primary determinant for catalytic activity on $\mathrm{Au}$ nanoparticle catalysts is the availability of low-coordinated gold atoms, which are also likely to be important for oxygen binding and activation ${ }^{27,45,46}$ The weak binding and activation of oxygen have long been considered keys to the high catalytic activity of supported $\mathrm{Au}$ catalysts, and there is a large body of experimental and theoretical literature suggesting that $\mathrm{O}_{2}$ adsorption likely occurs on nanoparticle corner or edge sites. ${ }^{2}$ This means that $\mathrm{O}_{2}$, which binds to $\mathrm{Au}$ much more weakly than $\mathrm{CO}$, has to compete for similar binding sites. The experimental finding that a substantial fraction of $\mathrm{CO}$ binding sites are unoccupied under reaction pressures makes this competition much more plausible.

Because oxygen binding to $\mathrm{Au}$ surfaces and nanoparticle catalysts is very weak, it has been extremely difficult to measure. We are aware of only one experimental measurement of the binding constant for $\mathrm{O}_{2}$ on $\mathrm{Au}$, a kinetic measurement extracted from the oxygen dependence of the catalytic rate during $\mathrm{CO}$ oxidation catalysis. ${ }^{27}$ This study found the $\mathrm{O}_{2}$ binding constants for the WGC and MPC catalysts to be similar (both were approximately $35 \mathrm{~atm}^{-1}$, which corresponds to a $\Delta H_{\mathrm{ads}} \approx-53$ $\mathrm{kJ} / \mathrm{mol}$ calculated in the same fashion as above). This value compares very favorably with the high-coverage (weaker) $\mathrm{CO}$ binding constants $\left(27 \mathrm{~atm}^{-1}\right.$, or $\left.\Delta H_{\mathrm{ads}} \approx-52 \mathrm{~kJ} / \mathrm{mol}\right)$ determined in the current study. The similarity of the high-coverage $\mathrm{CO}$ and $\mathrm{O}_{2}$ binding constants indicates that, even under differential reaction conditions where conversions are low and $\mathrm{CO}$ coverage is relatively high and constant, $\mathrm{O}_{2}$ can effectively compete with $\mathrm{CO}$ for the remaining open corner and edge sites. This provides another important explanation for the high activity of $\mathrm{Au} \mathrm{CO}$ oxidation catalysts: our study shows not only that $\mathrm{CO}$ binding sites are available under reaction conditions but also that the similarity between the high-coverage $\mathrm{CO}$ and $\mathrm{O}_{2}$ binding constants under these conditions means that $\mathrm{CO}$ and $\mathrm{O}_{2}$ will bind to the remaining adsorption sites with roughly equal affinity.

\section{Conclusions}

$\mathrm{CO}$ adsorption on three high surface area $\mathrm{Au} / \mathrm{TiO}_{2}$ catalysts was evaluated with infrared transmission spectroscopy under conditions typically used in $\mathrm{CO}$ oxidation catalysis. The three catalysts had essentially the same $\mathrm{CO}$ adsorption properties under the pressure range studied (0.05-10 Torr). Binding constants were extracted from the adsorption data using a twosite Langmuir model and a sliding-tangent fit to the Langmuir equation. Both models did a good job of describing the observed coverage dependence of the binding constant. Coveragedependent heat of adsorption values extracted from the binding constant data were consistent with measurements on planar model systems and under conditions somewhat outside the working conditions of the catalyst.

Comparing the high surface area catalysts with previous measurements on other systems suggests that the $\mathrm{CO}$ binding sites are likely to be highly uncoordinated corner and edge atoms. A key result from our studies is that roughly one-quarter of these $\mathrm{CO}$ binding sites remain open under $\mathrm{CO}$ oxidation catalysis conditions. Further, the high-coverage $\mathrm{CO}$ binding constants (or weak $\mathrm{CO}$ binding sites) are comparable to $\mathrm{O}_{2}$ binding constants measured during $\mathrm{CO}$ oxidation catalysis. This means that $\mathrm{O}_{2}$ can effectively compete with $\mathrm{CO}$ for the open corner and edge sites, providing an important experimental explanation for the high $\mathrm{CO}$ oxidation activity of $\mathrm{Au} / \mathrm{TiO}_{2}$ catalysts.

Acknowledgment. The authors gratefully acknowledge the U.S. National Science Foundation (Grant No. CHE-0449549) 
and the Robert A. Welch Foundation (Grant No. W-1552) for financial support of our work.

\section{References and Notes}

(1) Bond, G. C.; Louis, C.; Thompson, D. T. Catalysis by Gold; Imperial College Press: London, 2006; Vol. 6.

(2) Kung, M. C.; Davis, R. J.; Kung, H. H. J. Phys. Chem. C 2007, $111,11767-11775$.

(3) Fu, Q.; Saltsburg, H.; Flytzani-Stephanopoulos, M. Science 2003, $301,935-938$.

(4) Enache, D. I.; Edwards, J. K.; Landon, P.; Solsona-Espriu, B.; Carley, A. F.; Herzing, A. A.; Watanabe, M.; Kiely, C. J.; Knight, D. W.; Hutchings, G. J. Science 2006, 311, 362-365.

(5) Hughes, M. D.; Xu, Y.-J.; Jenkins, P.; McMorn, P.; Landon, P.; Enache, D. I.; Carley, A. F.; Attard, G. A.; Hutchings, G. J.; King, F.; Stitt, E. H.; Johnston, P.; Griffin, K.; Kiely, C. J. Nature 2005, 437, 11321135.

(6) Corma, A.; Gonzalez-Arellano, C.; Iglesias, M.; Sanchez, F. Angew. Chem., Int. Ed. 2007, 46, 7820-7822.

(7) Corma, A.; Serna, P. Science 2006, 313, 332-334.

(8) Boronat, M.; Concepcion, P.; Corma, A.; Gonzalez, S.; Illas, F.; Serna, P. J. Am. Chem. Soc. 2007, 129, 16230-16237.

(9) Corma, A.; Concepcion, P.; Serna, P. Angew. Chem., Int. Ed. 2007, 46, 7266-7269.

(10) Christensen, C. H.; Joergensen, B.; Rass-Hansen, J.; Egeblad, K.; Madsen, R.; Klitgaard, S. K.; Hansen, S. M.; Hansen, M. R.; Andersen, H. C.; Riisager, A. Angew. Chem., Int. Ed. 2006, 45, 4648-4651.

(11) Abild-Pedersen, F.; Andersson, M. P. Surf. Sci. 2007, 601, 17471753.

(12) Mason, S. E.; Grinberg, I.; Rappe, A. M. Phys. Rev. B: Condens. Matter Mater. Phys. 2004, 69, 161401-161404.

(13) Nieskens Davy, L. S.; Curulla-Ferre, D.; Niemantsverdriet, J. W. ChemPhysChem 2006, 7, 1075-1080.

(14) Rogal, J.; Reuter, K.; Scheffler, M. Phys. Rev. B: Condens. Matter Mater. Phys. 2007, 75, 205433.

(15) Zeinalipour-Yazdi, C. D.; Cooksy, A. L.; Efstathiou, A. M. Surf. Sci. 2008, 602, 1858-1862.

(16) Ponec, V.; Bond, G. C. Eds. Catalysis by Metals and Alloys; Elsevier: Amsterdam, 1995; Vol. 95.

(17) Oxford, S. M.; Henao, J. D.; Yang, J. H.; Kung, M. C.; Kung, H. H. Appl. Catal., A 2008, 339, 180-186.

(18) Menegazzo, F.; Manzoli, M.; Chiorino, A.; Boccuzzi, F.; Tabakova,

T.; Signoretto, M.; Pinna, F.; Pernicone, N. J. Catal. 2006, 237, 431-434

(19) Meier, D. C.; Goodman, D. W. J. Am. Chem. Soc. 2004, 126, 1892 1899.

(20) Zhao, Z.; Diemant, T.; Rosenthal, D.; Christmann, K.; Bansmann, J.; Rauscher, H.; Behm, R. J. Surf. Sci. 2006, 600, 4992-5003.

(21) Diemant, T.; Hartmann, H.; Bansmann, J.; Behm, R. J. J. Catal. 2007, 252, 171-177.

(22) Lemire, C.; Meyer, R.; Shaikhutdinov, S. K.; Freund, H. J. Surf. Sci. 2004, 552, 27-34.

(23) Yim, W.-L.; Nowitzki, T.; Necke, M.; Schnars, H.; Nickut, P.; Biener, J.; Biener, M. M.; Zielasek, V.; Al-Shamery, K.; Kluener, T. Baeumer, M. :J. Phys. Chem. C 2007, 111, 445-451.

(24) Meier, D. C.; Bukhtiyarov, V.; Goodman, D. W. J. Phys. Chem. B 2003, 107, 12668-12671.
(25) Kim, J.; Samano, E.; Koel, B. E. J. Phys. Chem. B 2006, 110 , $17512-17517$.

(26) Derrouiche, S.; Gravejat, P.; Bianchi, D. J. Am. Chem. Soc. 2004, 126, 13010-13015.

(27) Long, C. G.; Gilbertson, J. D.; Vijayaraghavan, G.; Stevenson, K. J.;

Pursell, C. J.; Chandler, B. D. J. Am. Chem. Soc. 2008, 130, 10103-10115.

(28) Korkosz, R. J.; Gilbertson, J. D.; Prasifka, K. S.; Chandler, B. D. Catal. Today 2007, 122, 370-377.

(29) $\mathrm{CO}$ adsorption on $\mathrm{TiO}_{2}$, which appeared as a peak near $2050 \mathrm{~m}^{-1}$ (wavenumber), was only observed at much lower temperatures, ca. below $253 \mathrm{~K}$

(30) Hakkinen, H.; Abbet, S.; Sanchez, A.; Heiz, U.; Landman, U. Angew. Chem., Int. Ed. 2003, 42, 1297-1300.

(31) Dumas, P.; Tobin, R. G.; Richards, P. L. Surf. Sci. 1986, 171, 579599.

(32) Bradshaw, A. M.; Pritchard, J. Proc. R. Soc. A 1970, 316, 169183.

(33) France, J.; Hollins, P. J. Electron Spectrosc. Relat. Phenom. 1993, 64-65, 251-258.

(34) Chen, M.; Cai, Y.; Yan, Z.; Goodman, D. W. J. Am. Chem. Soc. 2006, 128, 6341-6346.

(35) Spiewak, B. E.; Dumesic, J. A. Thermochim. Acta 1998, 312, 95104.

(36) Guerrero-Ruiz, A.; Maroto-Valiente, A.; Cerro-Alarcon, M.; Bachiller-Baeza, B.; Rodriguez-Ramos, I. Top. Catal. 2002, 19, 303-311.

(37) Phillips, J. J. Therm. Anal. 1997, 49, 541-552.

(38) Podkolzin, S. G.; Shen, J.; De Pablo, J. J.; Dumesic, J. A. J. Phys. Chem. B 2000, 104, 4169-4180.

(39) Shen, J.; Hill, J. M.; Watwe, R. M.; Podkolzin, S. G.; Dumesic, J. A. Catal. Lett. 1999, 60, 1-9.

(40) Xia, X.; Naumann d'Alnoncourt, R.; Muhler, M. :J. Therm. Anal. Calorim. 2008, 91, 167-172.

(41) Roze, E.; Gravejat, P.; Quinet, E.; Rousset, J. L.; Bianchi, D. J. Phys. Chem. C 2009, 113, 1037-1045.

(42) Manzoli, M.; Chiorino, A.; Boccuzzi, F. Surf. Sci. 2003, 532$535,377-382$.

(43) As a reviewer pointed out, the $\mathrm{CO}$ coverage under reaction conditions is more complicated. In a working reactor, $\mathrm{CO}$ coverage will depend on the conversion at any given time and will vary throughout the reactor bed. We, therefore, present the arguments regarding $\mathrm{CO}$ and $\mathrm{O}_{2}$ competition in the context of the limiting case of differential reaction conditions. Under these conditions, conversions are low $(<2 \%)$ and reactant pressures are essentially constant throughout the reactor bed. CO coverages are, therefore, constant and approximately equal to the inlet composition of the feed. This represents the "worst-case scenario" for $\mathrm{O}_{2}$ competition because the number of available binding sites is minimized. At higher conversions, the $\mathrm{CO}$ partial pressure (and, therefore, coverage) drops throughout the reactor, freeing up even more sites for $\mathrm{O}_{2}$ binding and activation. So, as long as the $\mathrm{CO}$ pressure is near or below $1 \% \mathrm{CO}$, the data indicate that a substantial fraction of the $\mathrm{CO}$ binding sites will be available for $\mathrm{O}_{2}$ binding.

(44) Falsig, H.; Hvolboek, B.; Kristensen, I. S.; Jiang, T.; Bligaard, T.; Christensen, C. H.; Noerskov, J. K. Angew. Chem., Int. Ed. 2008, 47, 48354839 .

(45) Lopez, N.; Janssens, T. V. W.; Clausen, B. S.; Xu, Y.; Mavrikakis, M.; Bligaard, T.; Norskov, J. K. J. Catal. 2004, 223, 232-235.

(46) Xu, Y.; Mavrikakis, M. J. Phys. Chem. B 2003, 107, 9298-9307. 